\title{
Paravalvular leak prediction after transcatheter aortic valve replacement with self-expandable prosthesis based on quantitative aortic calcification analysis
}

\author{
Agata Wiktorowicz ${ }^{1}$, Adrian Wit ${ }^{2}$, Krzysztof Piotr Malinowski ${ }^{1}$, Artur Dziewierz ${ }^{1}$, Lukasz Rzeszutko ${ }^{1}$, \\ Dariusz Dudek ${ }^{1}$, Pawel Kleczynski ${ }^{3} \wedge$ \\ ${ }^{1}$ Jagiellonian University Medical College, Faculty of Medicine, Institute of Cardiology, $2^{\text {nd }}$ Department of Cardiology, 30-688, Krakow, Poland; \\ ${ }^{2}$ Faculty of Physics and Applied Computer Science, AGH University of Science and Technology, 30-059 Krakow, Poland; Jagiellonian University \\ Medical College, Faculty of Medicine, Institute of Cardiology, Department of Interventional Cardiology, John Paul II Hospital, 31-202 Krakow, \\ Poland
}

Correspondence to: Pawel Kleczynski, MD, PhD, FESC. Department of Interventional Cardiology, Jagiellonian University Medical College, 80 Pradnicka St., 31-501 Krakow, Poland. Email: kleczu@interia.pl.

Background: Paravalvular leak (PVL) is one of the most common complications of transcatheter aortic valve replacement (TAVR) and affects short- and long-term outcomes. The aim of this study was to identify the computed tomography (CT) imaging biomarkers that allow PVL after TAVR to be predicted.

Methods: Patients were included who had severe aortic valve stenosis, had undergone TAVR with a selfexpanding valve, and had undergone a pre-procedural CT scan. Data on baseline characteristics, procedural and long-term outcomes were collected retrospectively. We used MATLAB software with a self-developed algorithm for CT scan analysis and found parameters that quantified aortic valve calcifications (AVC) in detail.

Results: Fifty patients were included. The identified CT-derived parameters included AVC size, volume, thickness and density, as well as calcium radial distribution. The volume of the largest calcium block, calcium perimeter and calcium size (assessed by Feret's diameter) showed a strong association with PVL occurrence after TAVR $(\mathrm{P}=0.012, \mathrm{P}=0.001$ and $\mathrm{P}=0.045$, respectively). The prognostic model showed that a $10 \mathrm{~mm}^{2}$ increase in the local AVC amount in each valve section was associated with a 9.8\% (95\% CI: 2-18\%; $\mathrm{P}=0.019$ ) increase in the risk of PVL occurrence in the corresponding area after TAVR. ROC analysis revealed that the cut-off point of the AVC area was $96.5 \mathrm{~mm}^{2}$ in the polar coordinate system presentation. Kaplan-Meier curves showed worse PVL-free survival in patients with more than $96.5 \mathrm{~mm}^{2}$ of calcium area $(\mathrm{P}=0.013$; log-rank).

Conclusions: Quantitative AVC assessment for PVL prediction may play an important role in screening before TAVR. In future, the use of quantitative AVC assessment as an imaging biomarker in TAVR candidates and the creation and extension of an online database containing quantitative AVC parameters may help to identify high PVL risk patients.

Keywords: Aortic stenosis (AS); calcifications; computed tomography (CT); paravalvular leak (PVL); transcatheter aortic valve replacement (TAVR)

Submitted May 16, 2020. Accepted for publication Sep 21, 2020.

doi: 10.21037/qims-20-669

View this article at: http://dx.doi.org/10.21037/qims-20-669

^ ORCID: 0000-0002-8591-8739. 


\section{Introduction}

The number of transcatheter aortic valve replacement (TAVR) procedures in patients with severe aortic valve stenosis (AS) is increasing every year, and the indications are being constantly extended (1). One of the most common complications after TAVR is paravalvular leak (PVL), which influences acute and long-term clinical outcomes $(2,3)$. The incidence of at least mild PVL after TAVR is estimated at ca. 20 to $60 \%$ of patients and occurs more frequently than in the case of surgical aortic valve replacement (AVR) $(4,5)$. During short- and long-term follow-up, previous studies have shown that the occurrence of moderate to severe PVL increases mortality after TAVR $(6,7)$. However, there is no unified method of identifying patients with increased PVL risk. Moreover, there is no clear, objective statement regarding perioperative management or proper valvular prostheses selection, both of which make it possible to reduce the postprocedural occurrence of PVL. The European Imaging Biomarkers ALLiance (EIBALL), which is a subcommittee of the European Society of Radiology (ESR), emphasized the important role of imaging biomarkers as they are able to quantify the extent of morphological characteristics. Also, the EIBALL subcommittee indicates the necessity of building imaging databases and their use in the prediction of clinical outcomes (8).

The purpose of our study was to identify imaging biomarkers to predict PVL occurrence after TAVR. We focused on a detailed analysis of aortic valve calcifications (AVC) in preprocedural multi-slice computed tomography (MSCT) of AS patients qualified for TAVR.

\section{Methods}

Fifty patients with severe AS (tricuspid aortic valve, aortic valve area $<1.0 \mathrm{~cm}^{2}$ and mean pressure gradient $>40 \mathrm{mmHg}$ ) who had undergone TAVR with a self-expanding valve (Evolut R, Medtronic Inc, USA) were included in the study. The valve size selection was based on the CT-derived aortic annulus size, together with perimeter according to valve sizing charts. Imaging data from preprocedural contrast-enhanced CT and postprocedural transthoracic echocardiography (TTE) were analyzed. Data on baseline characteristics, procedural and long-term outcomes were collected. All clinical and imaging data were collected and analyzed retrospectively. The study was approved by the Ethical Board of the Jagiellonian University Medical
College, Krakow, Poland (1072.6120.234.2017). The study protocol conforms to the ethical guidelines of the 1975 Declaration of Helsinki with later amendments. Written informed consent was obtained from the patient for publication of this study and any accompanying images. A copy of the written consent is available for review by the Editor-in-Chief of this journal.

All contrast-enhanced CT scans were performed using a Toshiba scanner (Toshiba Aquilion 64 CT scanner, Toshiba, Tokyo, Japan). All acquisitions were performed using an institutional standard protocol and reconstruction settings. The protocol involved a scan run that consisted of the prospective acquisition of contiguous transverse slices $(0.5 \mathrm{~mm} \times 64$ detector row) in a single breath-hold of 6-10 seconds, triggered at $60-80 \%$ of the electrocardiogram $\mathrm{R}$-to-R wave interval.

MSCT scans were analyzed twice: (I) by an experienced radiologist before TAVR, using the traditional method; (II) by two trained corelab analysts blinded to the clinical and echocardiographic data, using dedicated software based on MATLAB version R2017a (MathWorks, Natick, MA, USA) and ImageJ version 1.51 (NIH, USA) with the BoneJ plugin version 1.4.2 (9). The limited intraobserver and interobserver variability for AVC evaluation was confirmed by Intraclass Correlation Coefficient (ICC), which was 0.992 (95\% CI, 0.990-0.995) and 0.991 (95\% CI, 0.988-0.993), respectively.

Traditional CT parameters measured by radiologist were used to validate the quantitative parameters received by our dedicated software based on MATLAB.

TTE was performed on a GE Healthcare echocardiograph (Vivid E9, GE Healthcare, Chicago, Illinois, USA). A trained physician who was blinded to the clinical and radiological data analyzed the postprocedural TTE images to detect PVL occurrence in 1-, 3-, 6-, and 12-month follow-ups, and its severity was classified according to the Valve Academic Research Consortium-2 (VARC-2) Consensus Recommendation and American Society of Echocardiography guidelines for the evaluation of valvular regurgitation after percutaneous intervention $(10,11)$. We based on the assessment on semi-quantitative methods in color doppler echocardiography such as jet number, location, direction, and eccentricity and circumferential extent.

Clinical follow-up data, including novel medical incidents such as pacemaker implantation, were collected retrospectively at 1-, 3-, 6-, and 12-month observations. 

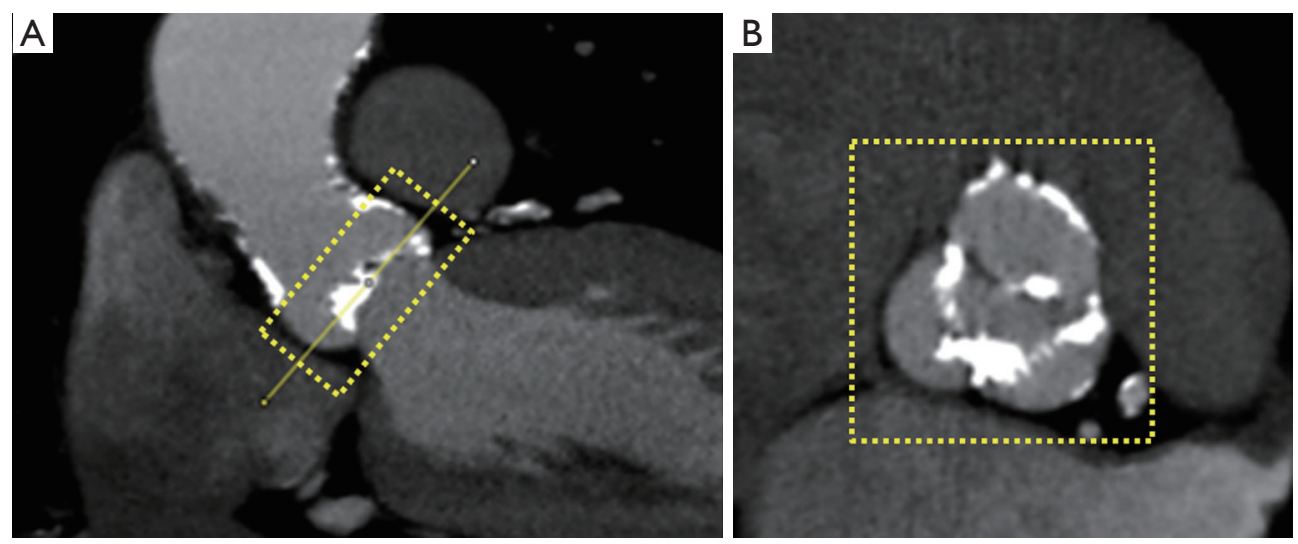

Figure 1 The coronal view (A) and perpendicular plane (B) for aortic valve. The images were rotated to obtain planes perpendicular to the aortic valve. The yellow dotted squares show the boundaries for VOI and ROI analysis. ROI, region of interest; VOI, volume of interest.

\section{CT-derived data processing}

All calculations were performed in the Volume-Of-Interest (VOI), which is defined as a stack of closed planar polygons of CT slices; this is a $3 \mathrm{D}$ space which in our case contains the calcified aortic valve (between the highest visible point of the aortic apparatus and the left ventricle outflow tract). For better visualization of calcified aortic leaflets, the initial imaging data underwent computational modelling, recomputation and rotation into a set of cross-sections parallel to the plane of the valve ring and short axis of the ascending aorta. The result of computational modelling is presented in Figure 1.

The threshold cut-off value for detection of calcifications was set individually for each case, but it was typically set in the range 391-689 Hounsfield units (HU). This threshold was adopted due to the contrast medium attenuation in the aortic root. Every calcified mass except for the AVC was excluded from the analysis.

The selected VOI was then used to adjust the corresponding planar projection [region-of-interest (ROI)] of the aortic valve using the maximum intensity of the calcium conglomerates contained in the VOI. However, the large variability of calcium distribution causes a quantification problem in a single-slice image (before processing). To solve this problem and to provide better visualization of local AVC distribution, radial AVC presentation was implemented. The ROI presentation was inscribed in a circular plane with a central point that was previously mathematically assigned as the center point of the aortic valve. The AVC amount was individually calculated from the mass center in 1-degree slices until the entire circle had been analyzed. This approach allowed the exact local AVC distribution to be presented, both in the direct CT image and in the form of a chart. An exemplary image is presented in Figure 2, which shows the radial image intensity ( $y$-axis) in reference to degree coordination ( $x$-axis).

\section{CT-derived parameters}

Technical aspects of CT data acquisition and AVC quantification have been described in detail previously $(12,13)$. The AVCs were presented and analyzed in a twofold manner:

(I) AVC was presented as a whole mass in the stenotic valve. In this category, the analyzed parameters were assigned to two groups: morphometric (AVC size, volume and thickness in ROI and VOI presentation) and textural (AVC radiodensity).

(II) AVC was presented in a radial presentation. This category presents AVC orientation and distribution (topological parameters); the local AVC amount was described in each of the three sections of the valve. The aortic valve ROI projection was presented on the circular plane and was subdivided into three zones, each containing 1201 -degree slices (Figure 3A). The AVC amount was calculated radially from the stenotic valve mass center for each 1-degree slice, and the total in each of the three sections was then calculated. The AVC amount is presented in square millimeters $\left(\mathrm{mm}^{2}\right)$.

The presentation of the AVC amount in each of the three valve ROI sections allowed us to compare the local 


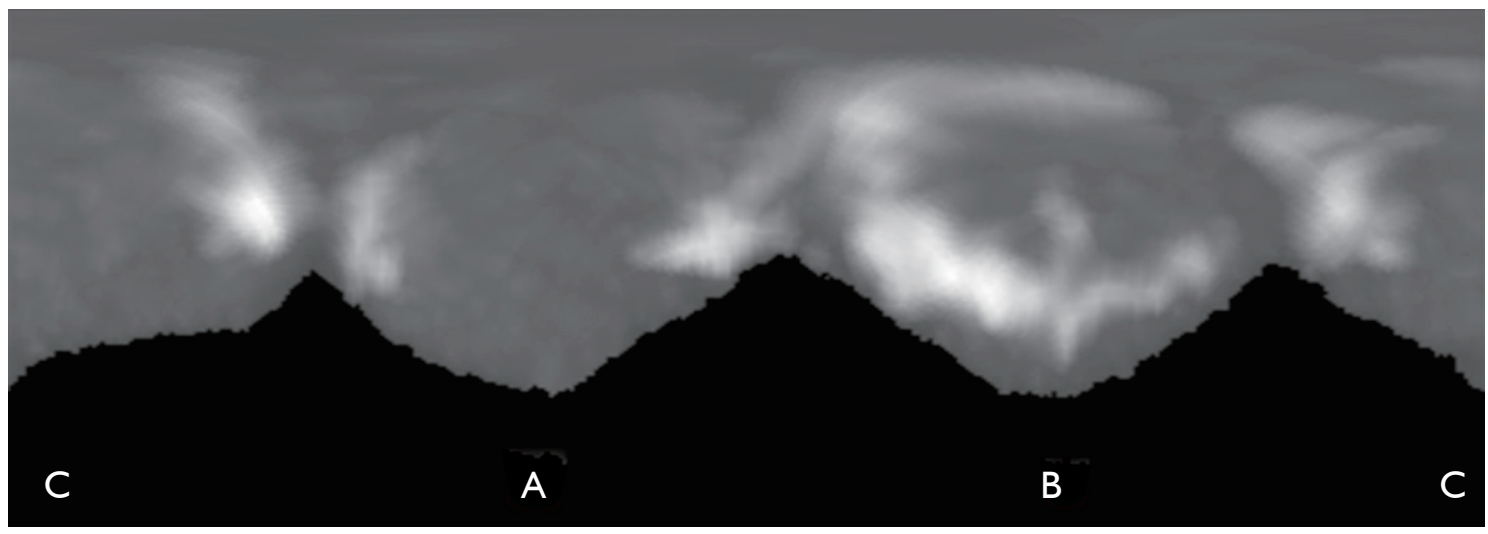

Figure 2 Radial presentation of the aortic valve. Visualization of the polar coordinate system makes it possible to present the degree of calcification on a single aortic leaflet $(\mathrm{A}, \mathrm{B}, \mathrm{C})$.
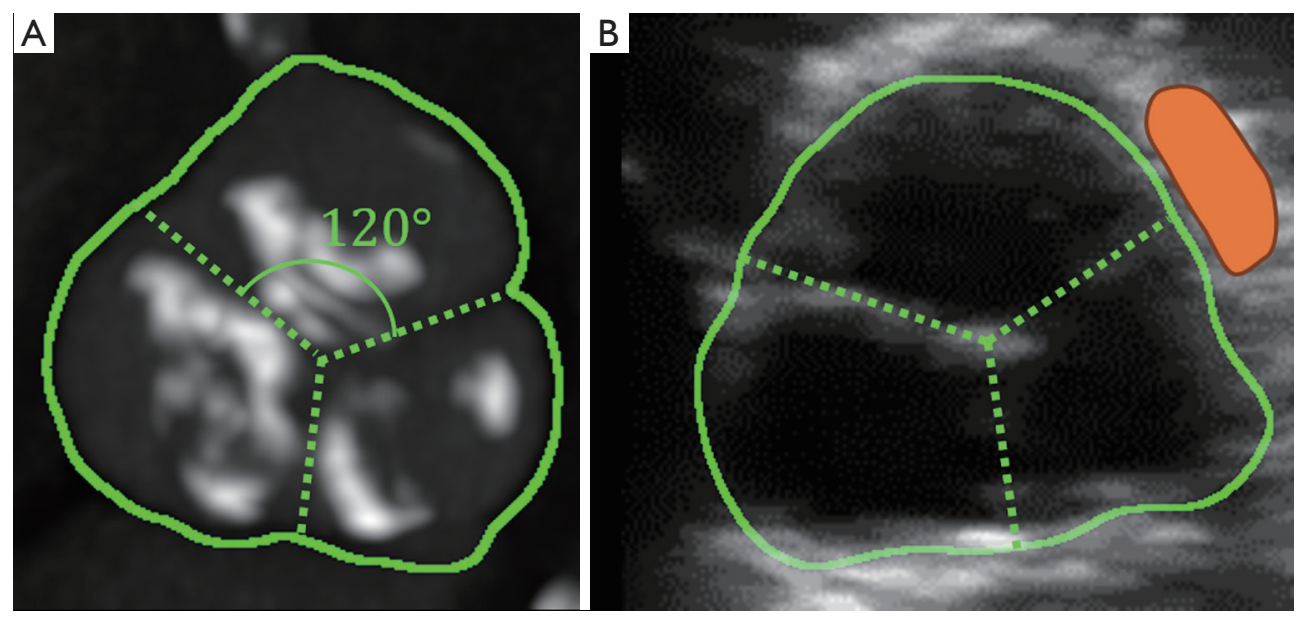

Figure 3 Radial calcium presentation: (A) ROI presentation divided into three zones (120 degrees each) and radial summation of AVC amount in each zone (explanation in the text); (B) TTE image with PVL location. AVC, aortic valve calcification; PVL, paravalvular leak; ROI, region of interest; TTE, transthoracic echocardiography.

AVC amount and the location of the PVL site after TAVR (if it occurred) (Figure 3B). Additionally, 3D AVC models with a color scale depending on the degree of calcium thickness were obtained (Figure 4).

A single case analysis using our method of AVC quantification took about 20 minutes.

The cover index was calculated from the following formula:

$$
C I=\frac{P_{d}-A_{d}}{P_{d}} \times 100
$$

where CI = cover index $(-), P_{d}=$ prosthesis diameter $(\mathrm{mm})$, $A_{d}=$ annulus diameter $(\mathrm{mm})$,
Ellipticity and eccentricity indexes were calculated in three ways: based on traditional CT measurements; based on separate quantitative AVC calculations with ROI diameter and Feret's diameter. The ellipticity index was defined as the annulus maximum/minimum diameter. The eccentricity index was defined as 1 minus the annulus minimal diameter/maximal diameter.

\section{Statistical analysis}

Categorical variables were expressed as numbers (percentages). Continuous variables were expressed as mean \pm standard deviation or median (interquartile range). 

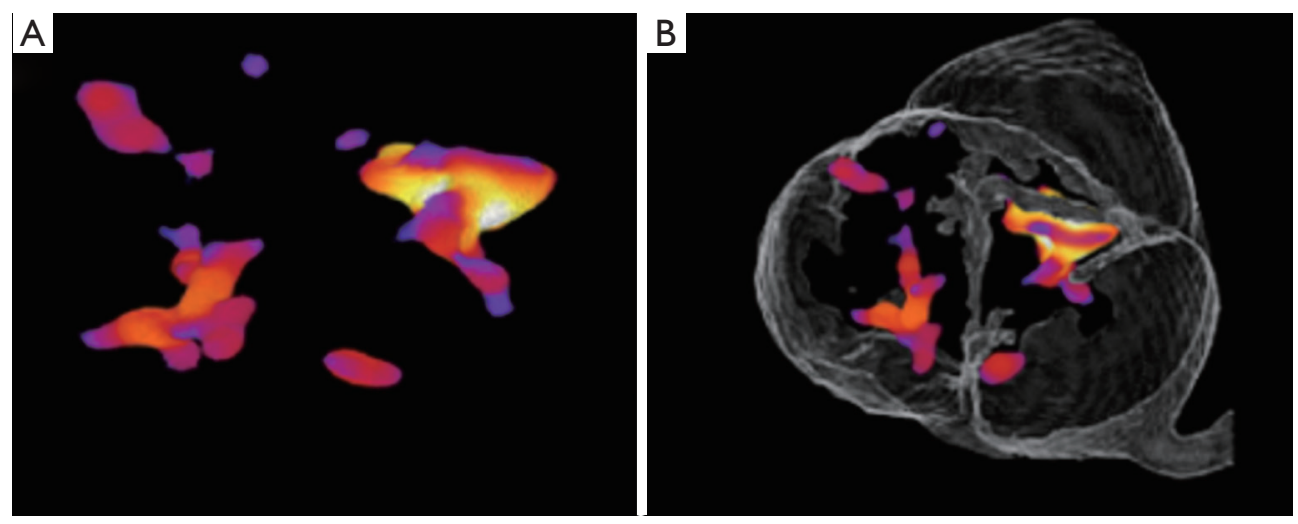

Figure 4 Exemplary AVC 3D models with a color scale representing calcium thickness. The brightest color is the thickest AVC part. (A) Separated AVC 3D models; (B) 3D models of AVC and its location in the aortic valve. 3D, 3 dimensional; AVC, aortic valve calcification.

The risk of PVL due to the AVC amount was assessed using the Cox method. The best multivariable model was chosen using stepwise combined regression with the Akaike information criterion as the target, with calcium locked in the model and adjusted for gender, body mass index and medical history. Risk ratios were presented with $95 \%$ confidence intervals. In the radial presentation analysis, the Cox regression model was constructed using one valve section as an observation and additional clustering of sections was considered. Bootstrap validation with 1,000 simulations was performed on the multivariable model with C-statistics as a measure of goodness of fit. ROC analysis was performed to calculate the cut-off point with the most discriminative power, then Kaplan-Meier curves with $95 \%$ CI were plotted for cases below and above the cut-off point, which were then compared using the logrank test. To assess the agreement between traditional MSCT measurement and AVC quantitative assessment, interobserver repeatability calculations were performed using Bland-Altman analysis. All tests were 2-tailed, and a $\mathrm{P}$ value $<0.05$ was considered statistically significant. All statistical analyses were performed using JMP 14.3 (SAS Institute Inc., Cary, NC, USA, 2019) and R 3.5.3 (R Foundation for Statistical Computing, Vienna, Austria, 2019).

\section{Results}

\section{Baseline and follow up data}

Table 1 shows the baseline characteristics and baseline echocardiographic and CT data (measured using the traditional method) of the study population.

During TAVR procedure, three patients required balloon postdilatation $(6 \%)$, which was performed in case of PVL 2 or more or in case of prosthesis underexpansion as assessed in TTE immediately after valve deployment. No periprocedural life-threatening complications (cardiac arrest, cardiac tamponade, severe arrhythmias) were observed. Five patients required pacemaker implantation within a year of their TAVR procedures (10\%). In 23 patients (46\%), PVL occurred during long-term followup: 13 patients had PVL grade 1 (26\%), 8 patients had PVL grade 2 (16\%), and 2 patients had PVL grade $3(4 \%)$.

\section{AVC quantitative parameters}

Measured using our method, the quantitative AVC parameters of the study population are shown in Table 2.

The univariate model shows significant association between the volume of the largest calcium block (VOI presentation), calcium perimeter and Feret's diameter (14) (ROI presentation), and PVL occurrence after TAVR. There was no significant correlation between the remaining ROI and VOI AVC parameters and long-term perivalvular complications after TAVR (pacemaker implantation and PVL occurrence) (Table 3).

\section{Radial presentation-PVL prognostic model and cross- validation of the results}

The multivariable model shows that AVC is an independent PVL predictor (Table 4). A $10 \mathrm{~mm}^{2}$ increase in local AVC amount in each section was associated with a $9.8 \%(95 \%$ 
Table 1 Baseline characteristics, baseline echocardiographic and computed tomography data

\begin{tabular}{lc}
\hline Variable & Outcome \\
\hline Baseline characteristics & \\
Age, median (IQR) (years) & $83.0(79.0-86.0)$ \\
Age $\geq 80$ years (\%) & 71.8 \\
Women (\%) & 71.8 \\
BMI, median (IQR) (kg/m²) & $26.8(24.8-29.0)$ \\
PCI before TAVR (\%) & 64.1 \\
CABG before TAVR (\%) & 15.4 \\
DM (\%) & 33.3 \\
DM on insulin (\%) & 10.3 \\
Arterial hypertension (\%) & 92.3 \\
Hyperlipidemia (\%) & 56.4 \\
CKD (\%) & 25.6 \\
Baseline echocardiographic and CT* data & \\
Mean AVA, median (IQR) (cm²) & $0.6(0.6-0.8)$ \\
Mean PG max, median (IQR) (mmHg) & $75.0(56.0-98.0)$ \\
Mean PG mean, median (IQR) (mmHg) & $47.0(33.0-61.0)$ \\
Mean EF, median (IQR) (\%) & $60.0(40.0-65.0)$ \\
Minimal valve annulus diameter in CT, & $23.0(21.0-25.0)$ \\
Maximal valve annulus diameter in CT, & \\
median (IQR) (mm) & $28.0(26.5-31.0)$ \\
\hline Mean cover index (\%) & \\
\hline
\end{tabular}

*, CT data based on traditional measurements. BMI, body mass index; PCl, percutaneous coronary intervention; CABG, coronary artery bypass; TAVR, transcatheter aortic valve replacement; $\mathrm{DM}$, diabetes mellitus; CKD, chronic kidney disease; AVA, aortic valve area; PG, pressure gradient; EF, ejection fraction; CT, computed tomography.

CI: $2 \%-18 \% ; \mathrm{P}=0.019)$ increase in the risk of PVL after TAVR in the corresponding area.

Bootstrap validation with 1,000 simulations was performed on the multivariable model with $\mathrm{C}$-statistics as a measure of goodness of fit. C-statistics was 0.7 , which indicates a good model (Bootstrap validation revealed C-statistic of 0.6).

ROC analysis revealed a cut-off point of $96.5 \mathrm{~mm}^{2}$ of the AVC amount assessed in radial slices. Kaplan-Meier curves showed the lowest PVL-free survival in patients with more than $96.5 \mathrm{~mm}^{2}$ of calcium $(\mathrm{P}=0.013$; log-rank) (Figure 5$)$.
Table 2 Aortic valve calcification quantitative parameters

\begin{tabular}{|c|c|}
\hline Variable & Outcome \\
\hline \multicolumn{2}{|l|}{ Morfometric AVC parameters } \\
\hline \multicolumn{2}{|l|}{ The volume of interest ( $\mathrm{VOI})^{*}$} \\
\hline $\begin{array}{l}\text { Calcium volume, median } \\
(\mathrm{IQR})\left(\mathrm{mm}^{3}\right)\end{array}$ & $613.3(345.3-1184.8)$ \\
\hline $\begin{array}{l}\text { Mean calcium thickness, median } \\
(\mathrm{IQR})(\mathrm{mm})\end{array}$ & $3.0(2.8-3.6)$ \\
\hline $\begin{array}{l}\text { Maximal calcium thickness, } \\
\text { median }(\mathrm{IQR})(\mathrm{mm})\end{array}$ & $4.6(4.0-5.6)$ \\
\hline $\begin{array}{l}\text { Number of separate calcium } \\
\text { blocks, median (IQR) (-) }\end{array}$ & $4.0(3.0-6.0)$ \\
\hline $\begin{array}{l}\text { The largest calcium block } \\
\text { volume, median }(\mathrm{IQR})\left(\mathrm{mm}^{3}\right)\end{array}$ & $334.0(197.9-729.8)$ \\
\hline \multicolumn{2}{|l|}{ The region of interest $(\mathrm{ROI})^{\star \star}$} \\
\hline $\mathrm{ROI}$ area, median (IQR) $\left(\mathrm{mm}^{2}\right)$ & $826.6(695.0-1041.4)$ \\
\hline $\begin{array}{l}\text { ROI perimeter, median } \\
(\mathrm{IQR})(\mathrm{mm})\end{array}$ & $114.3(103.5-129.5)$ \\
\hline $\begin{array}{l}\text { Calcium area, median } \\
(\mathrm{IQR})\left(\mathrm{mm}^{2}\right)\end{array}$ & $267.3(148.5-324.3)$ \\
\hline $\begin{array}{l}\text { Calcium perimeter, median } \\
(\mathrm{IQR})(\mathrm{mm})\end{array}$ & $168.8(140.2-202.1)$ \\
\hline $\begin{array}{l}\text { Maximal Feret diameter, median } \\
(\mathrm{IQR})(\mathrm{mm})\end{array}$ & $36.8(33.7-42.3)$ \\
\hline $\begin{array}{l}\text { Minimal Feret diameter, median } \\
(\mathrm{IQR})(\mathrm{mm})\end{array}$ & $30.7(28.0-35.2)$ \\
\hline \multicolumn{2}{|l|}{ Textural parameters } \\
\hline $\begin{array}{l}\text { Minimal calcium radiodensity, } \\
\text { median }(\mathrm{IQR})(\mathrm{HU})\end{array}$ & $534.0(454.0-592.0)$ \\
\hline $\begin{array}{l}\text { Maximal calcium radiodensity, } \\
\text { median (IQR) }(\mathrm{HU})\end{array}$ & $1370.0(1280.0-1506.0)$ \\
\hline $\begin{array}{l}\text { Mean calcium radiodensity, median } \\
(\mathrm{IQR})(\mathrm{HU})\end{array}$ & $788.5(714.8-854.3)$ \\
\hline \multicolumn{2}{|l|}{ Radial presentation } \\
\hline Field $A$, median $(\mathrm{IQR})\left(\mathrm{mm}^{2}\right)$ & $64.9(35.4-111.3)$ \\
\hline Field $B$, median $(\mathrm{IQR})\left(\mathrm{mm}^{2}\right)$ & $78.4(40.6-123.4)$ \\
\hline Field $\mathrm{C}$, median $(\mathrm{IQR})\left(\mathrm{mm}^{2}\right)$ & $104.4(79.0-133.1)$ \\
\hline
\end{tabular}

${ }^{*}$, refers to AVC volumetric parameters; ${ }^{\star \star}$, refers to planar AVC projection (2D image). HU, Hounsfield units.

Figure 6 shows 3D models and AVC quantification in the radial presentation of three exemplary cases. In case 1 , the AVC amount in each field did not exceed the threshold. In 
Table 3 Univariate analysis of the impact of aortic valve calcifications characteristics on paravalvular leak occurrence after transcatheter aortic valve replacement

\begin{tabular}{|c|c|c|c|c|}
\hline Variable & Odds ratio & $\mathrm{LCl}$ & $\mathrm{UCl}$ & $P$ value \\
\hline Calcium volume $\left(\mathrm{mm}^{3}\right)$ & 1.0000 & 1.0000 & 1.0000 & 0.19 \\
\hline Mean calcium thickness (mm) & 1.1632 & 0.4701 & 2.8779 & 0.74 \\
\hline Maximal calcium thickness (mm) & 1.0909 & 0.6844 & 1.7389 & 0.71 \\
\hline The largest calcium block volume $\left(\mathrm{mm}^{3}\right)$ & 1.0009 & 1.0002 & 1.0016 & 0.012 \\
\hline \multicolumn{5}{|l|}{ The region of interest $(\mathrm{ROI})^{\star \star}$} \\
\hline Feret's diameter (mm) & 1.1292 & 1.0569 & 1.2065 & 0.001 \\
\hline Calcium area $\left(\mathrm{mm}^{2}\right)$ & 1.0025 & 0.9993 & 1.0057 & 0.12 \\
\hline Maximal calcium density (HU) & 1.0014 & 0.9986 & 1.0041 & 0.34 \\
\hline Minimal calcium density (HU) & 1.0008 & 0.9960 & 1.0056 & 0.74 \\
\hline
\end{tabular}

${ }^{*}$, refers to AVC volumetric parameters; ${ }^{* *}$, refers to planar AVC projection (2D image). HU, Hounsfield units; mm, millimeters; mm ${ }^{2}$, square millimeters; $\mathrm{mm}^{3}$, cubic millimeters.

Table 4 Multivariable model for local paravalvular leak occurrence in radial presentation

\begin{tabular}{|c|c|c|c|c|c|}
\hline Variable & $\operatorname{Exp}($ coef) & $\operatorname{Exp}(-$ coef $)$ & Lower 95\% & Upper 95\% & $P$ value \\
\hline Balloon predilatation & 0.3663 & 2.7302 & 0.1282 & 1.047 & 0.06 \\
\hline Age per 1 year & 0.9425 & 1.0611 & 0.8990 & 0.988 & 0.014 \\
\hline BMI per $1 \mathrm{~kg} / \mathrm{m}^{2}$ & 1.0266 & 0.9741 & 0.8591 & 1.227 & 0.77 \\
\hline PCI before TAVR & 0.9904 & 1.0097 & 0.3135 & 3.129 & 0.99 \\
\hline
\end{tabular}

AVC, aortic valve calcifications; BMI, body mass index; DM, diabetes mellitus; PCI, percutaneous coronary intervention; TAVR, transcatheter aortic valve replacement.

this case, PVL formation was not observed at the 12-month follow-up. In cases 2 and 3, the AVC amount exceeded the threshold in field C in case 2, and in fields B and C in case 3. In these cases, PVL was observed at the 6-month TTE follow-up in locations corresponding to these fields in CT.

Additionally, we assessed the agreement between traditional CT measurement and AVC quantitative assessment of ellipticity and eccentricity indexes based on ROI diameter and Feret's diameter (Table 5 and Figure 7). Figure 8 shows Bland-Altman plots for comparison of traditional CT measurements and AVC quantitative assessment. For ellipticity index assessment, the mean relative differences were $-2.99 \%(-6.93-0.96 \%)$ and $-0.50 \%(-4.28-3.28 \%)$, respectively. For the eccentricity index, the mean relative differences were $-16.11 \%(-31.08 \%$ to $-1.14 \%)$ and $-4.64 \%(-18.43 \%$ to $9.14 \%)$, respectively.

\section{Discussion}

Preoperative CT assessment of patients with severe AS who have qualified for TAVR is obligatory and enables accurate procedure planning and correct device selection. 
CT assessment makes it possible to reduce the number of postoperative complications such as the occurrence of PVL (15-20). Severe AVC is one of the factors that makes PVL

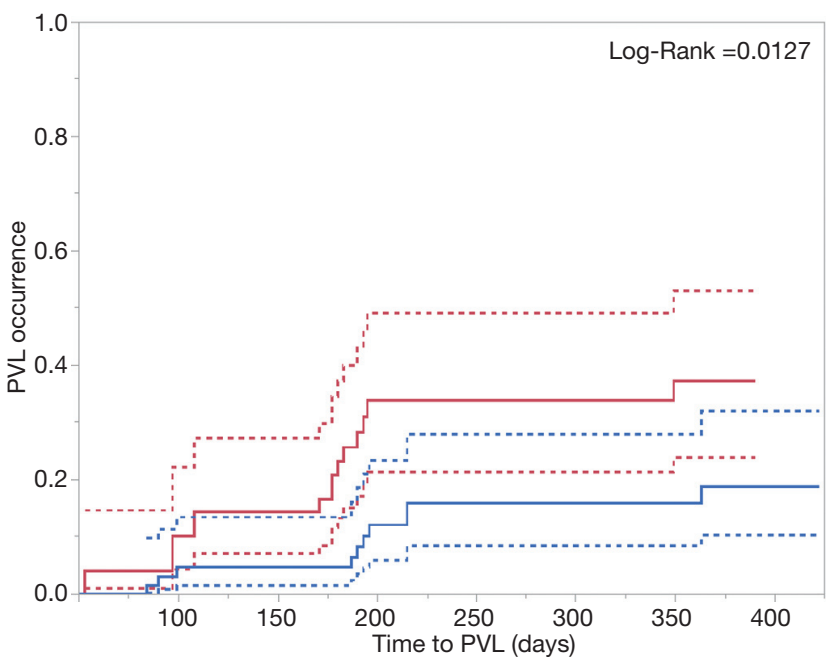

Figure 5 Kaplan-Meier curves for PVL occurrence during longterm follow-up, stratified by AVC (radial presentation). Blue: AVC amount $<96.5 \mathrm{~mm}^{2}$; red: AVC amount $\geq 96.5 \mathrm{~mm}^{2}$; dashed lines represent $95 \%$ CI. AVC, aortic valve calcification; PVL, paravalvular leak. more likely after TAVR, which is why it is obligatory to assess AVC degree in CT (21-23).

Pre-TAVR AVC assessment in CT is based on a semiquantitative method (Agatston score) or subjective visual assessment of CT data. For now, there is no prognostic model and no consensus on a specific imaging biomarker that would allow for unambiguous, objective identification of patients with a high risk of PVL. The authors of only a few previous studies have attempted to establish an objective, quantitative methodology of AVC description $(24,25)$. Single reports have indicated the role of AVC distribution in local PVL formation $(26,27)$. On the other hand, these reports show a lack of a harmonized, objective AVC assessment method.

We present a unique method of objective AVC assessment and its usefulness in patients who have qualified for TAVR. By building a database of quantitative AVC parameters, we specify parameters that allow estimation of high PVL formation after TAVR (largest calcium block volume, calcium perimeter, Feret's diameter). Additionally, radial presentation makes it possible to identify the exact location of higher probability of PVL occurrence in relation to the valve ring.

Initial assessment shows that our method has very good agreement with traditional CT measurements. Additionally,
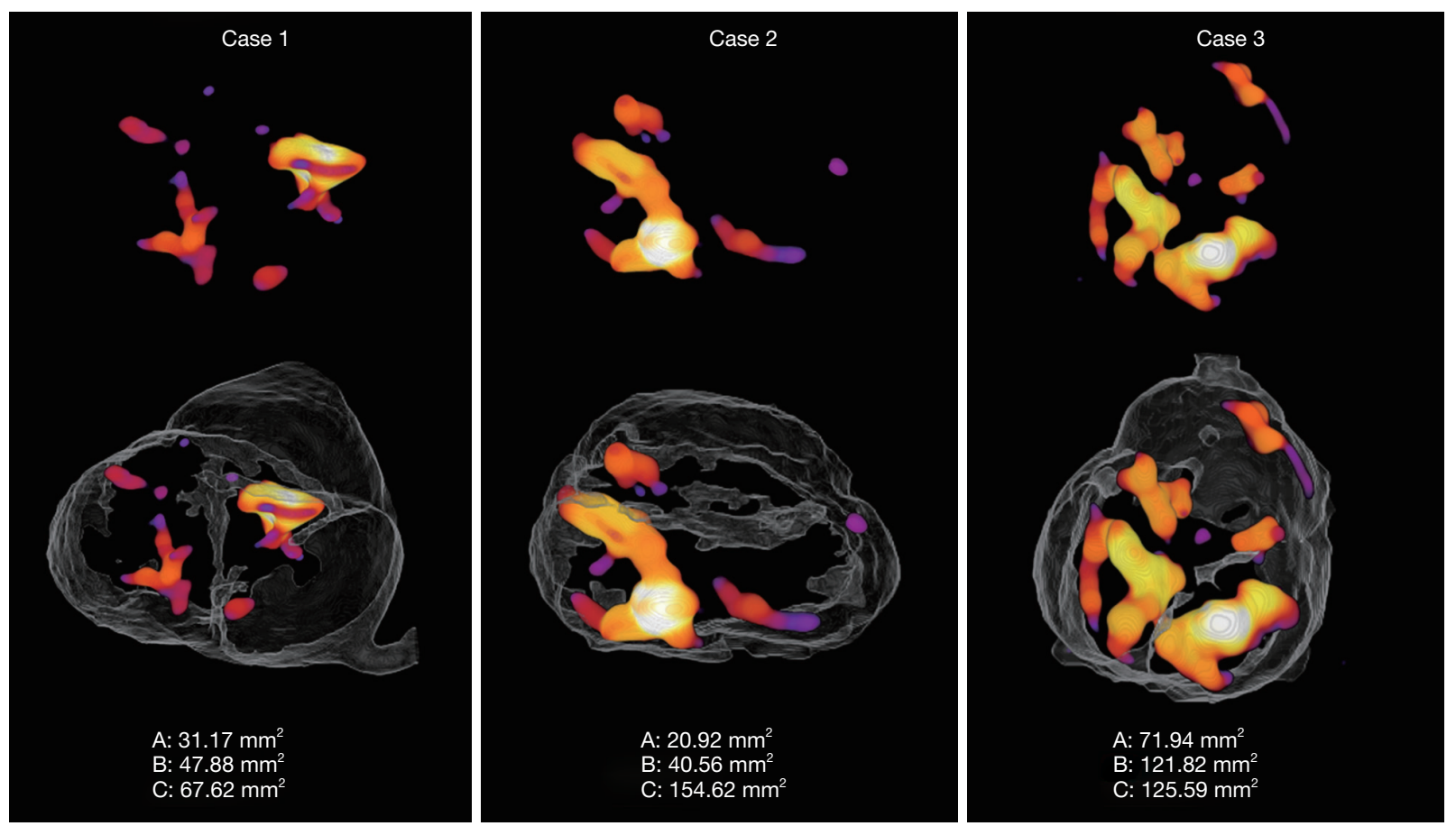

Figure $63 \mathrm{D}$ models and AVC quantification in three cases (explanation in the text). 3D, 3-dimensional; AVC, aortic valve calcification. 
Table 5 Mean ellipticity and eccentricity indexes measured in traditional computed tomography assessment and aortic valve calcifications quantitative assessment with the use of MATLAB

\begin{tabular}{lccc}
\hline \multirow{2}{*}{ Variable } & Traditional CT & AVC quantitative assessment in software based of MATLAB \\
\cline { 3 - 4 } & assessment & $\begin{array}{c}\text { Maximal and minimal } \\
\text { diameters measured in ROI }\end{array}$ & $\begin{array}{c}\text { Maximal and minimal diameter expressed } \\
\text { as maximal and minimal Feret's diameter }\end{array}$ \\
\hline Mean ellipticity index $(95 \% \mathrm{Cl})$ & $1.23(1.20-1.26)$ & $1.19(1.15-1.23)$ & $1.22(1.18-1.26)$ \\
Mean eccentricity index $(95 \% \mathrm{Cl})$ & $0.18(0.16-0.20)$ & $0.15(0.12-0.18)$ & $0.17(0.15-0.20)$ \\
\hline
\end{tabular}

AVC, aortic valve calcifications; CT, computed tomography; ROI, region of interest.
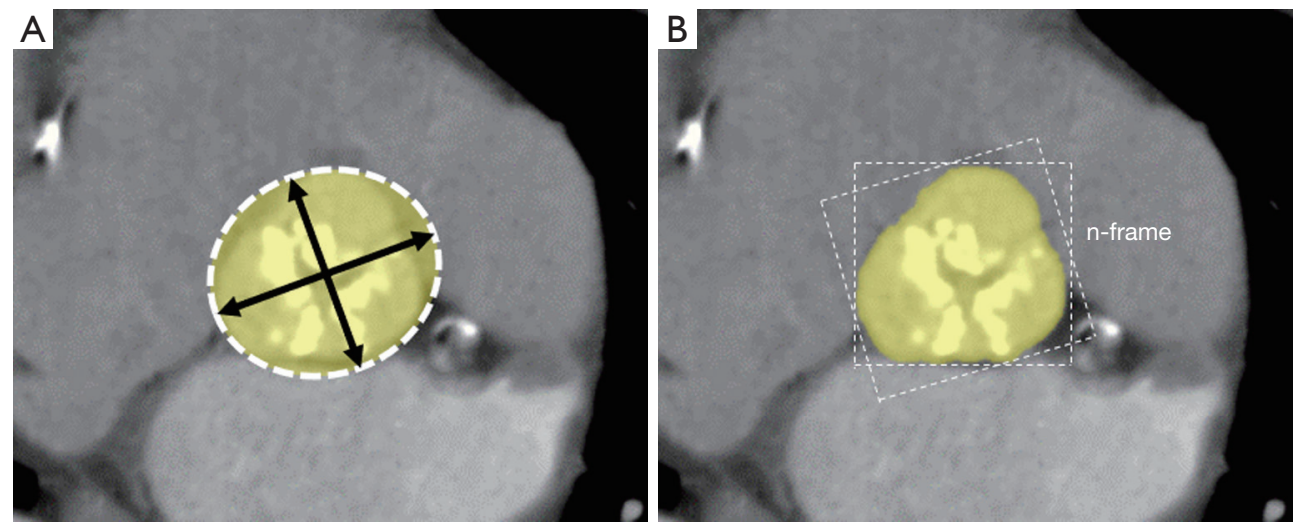

Figure 7 The AVC measurements using the traditional method (A) and the Feret's method (B). In the standard approach (A), the maximum and minimum ROI diameter are determined based on the individual judgment of the analyst, as the diameters of the most fitted circle. In the Feret's method (B), results are selected from maximal and minimal length of the rectangle fitted in different angles. This method ensures the repeatability and the high accuracy. AVC, aortic valve calcifications; ROI, region of interest.

a single case analysis is quick, thus it is possible to implement our method in real-time assessment. The predictive model for PVL occurrence based on imaging and clinical data is cross validated.

In the future, our work may affect diagnostic and therapeutic pathways in patients qualified for TAVR. Firstly, based on mathematical data, we will be able to list a group of patients with an increased likelihood of PVL formation. This group of patients will require increased observation both in the perioperative period and in longterm follow-ups. The first difference in the rate of PVL formation between patients with an AVC amount below and above $96.5 \mathrm{~mm}^{2}$ was noticed after just 1 month of follow-up. This could be very important information for physicians to do the first TTE within one month of TAVR for patients with higher AVC. Secondly, localization of the problematic area of the valve may have an impact on valve prosthesis selection. Additionally, the combination of radial presentation data with the more frequently described personalized simulations of the TAVR procedure (28-31) may affect both device selection and design.

Considering the increase in the popularity of TAVR, the relatively high PVL occurrence after TAVR, and the increase in mortality in patients with moderate to severe PVL, careful assessment of PVL predictors is required. In the future, the use of quantitative AVC parameters as PVL imaging biomarkers in TAVR patients may help to identify high PVL risk patients and may have an impact on device design and selection. These may impact the planning of the therapeutic and control path before and after TAVR in high PVL risk patients. To achieve these goals, the creation and extension of an online database containing quantitative AVC parameters is needed.

\section{Study limitations}

The main limitation of our study is the relatively small sample size and the lack of postprocedural PVL evaluation 

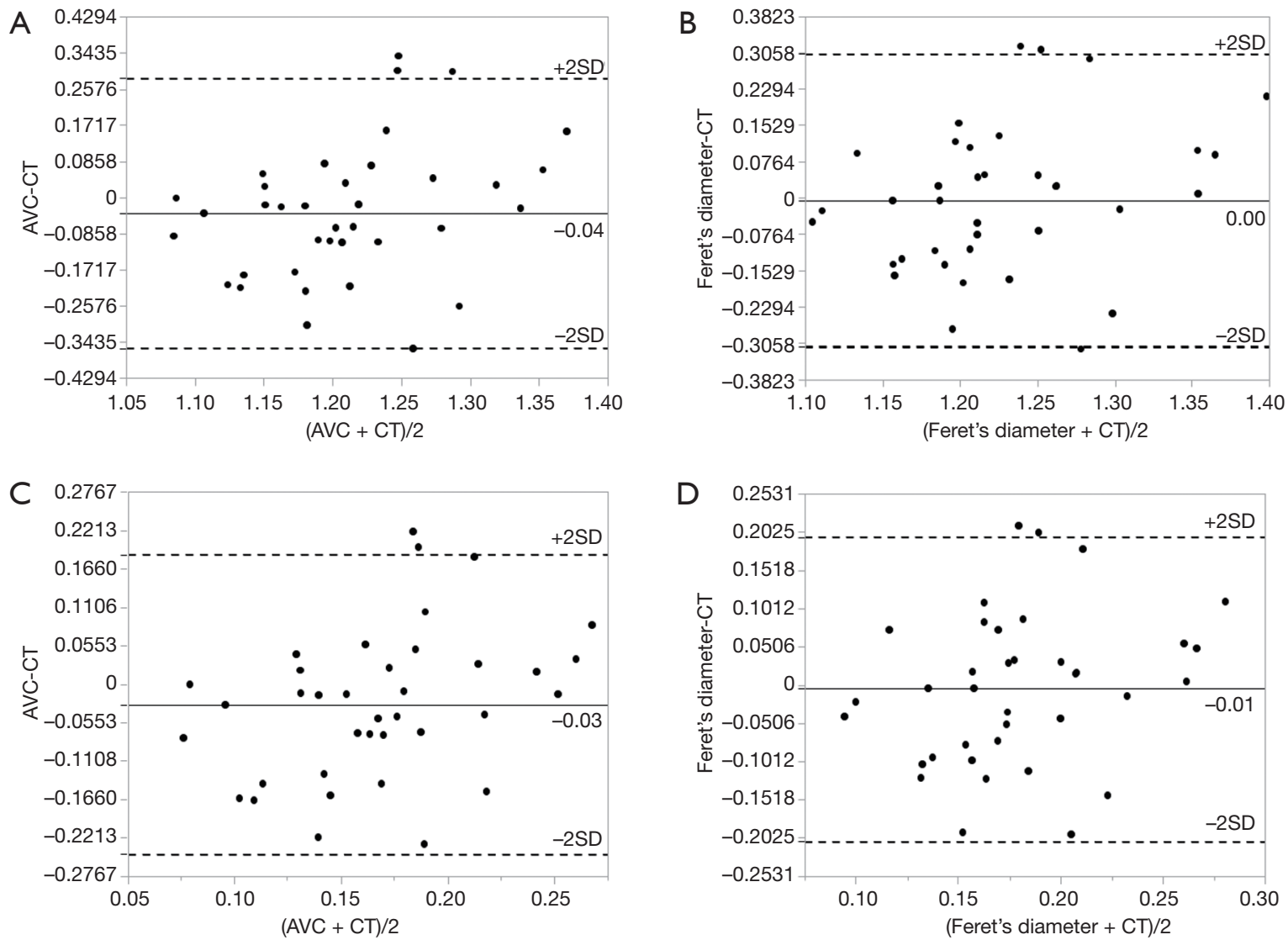

Figure 8 Bland-Altman plots for ellipticity index (A,B) and eccentricity index (C,D). AVC ellipticity and eccentricity indexes calculated on the basis of ROI maximal and minimal diameter; CT ellipticity and eccentricity indexes calculated on the basis of traditional CT measurements; Feret's diameter - ellipticity/eccentricity indexes calculated on the basis of ROI and Feret's minimal and maximal diameter. AVC, aortic valve calcification; CT, computed tomography; ROI, region of interest.

in transesophageal echocardiography, which is a reference method. Another limitation is the lack of the exact date of PVL formation as the patients were followed up on prescheduled dates on which only the PVL was revealed.

Most of the literature data regarding aortic valve calcium score refer to measurements based on non-contrast CT scans, which should be the reference method for AVC assessment. The quality of contrast enhanced CTs results from the interaction of multiple variables related to patient, scanner and contrast medium. Unlike non-contrast scans, the threshold for calcium detection on contrast-enhanced CT scans has not been standardized in the published reports. Both fixed-intensity values as well as dynamic thresholds related to luminal attenuation in the ascending aorta have been suggested (32). In our study, the threshold cut-off value was set individually (391-689 HU) for each case and was adopted due to contrast medium attenuation in the aortic root. Aortic valvular calcium score assessment based on contrast-enhanced CT is not a recommended method, but we chose to perform our measurements on these data because of their prevalence in TAVR patients. Contrast-enhanced CT is performed during preoperative assessment of every TAVR patient, and the use of this data for AVC evaluation carries no risk of additional radiation for these patients. Additionally, some studies have shown that AVC assessment in contrast-enhanced CT has good agreement with non-contrast MSCT (33).

\section{Conclusions}

Detailed, quantitative AVC assessment for PVL prediction may play an important role in screening before TAVR. In future, the use of quantitative AVC assessment as an imaging biomarker in TAVR candidates and the creation 
and extension of an online database containing quantitative AVC parameters may help to identify high PVL risk patients and may affect the therapeutic and diagnostic pathways of AS patients.

\section{Acknowledgments}

Funding: None.

\section{Footnote}

Conflicts of Interest: All authors have completed the ICMJE uniform disclosure form (available at http://dx.doi. org/10.21037/qims-20-669). The authors have no conflicts of interest to declare.

Ethical Statement: The study was approved by the Ethical Board of the Jagiellonian University Medical College, Krakow, Poland (1072.6120.234.2017). The study protocol conforms to the ethical guidelines of the 1975 Declaration of Helsinki with later amendments. Written informed consent was obtained from the patient for publication of this study and any accompanying images. A copy of the written consent is available for review by the Editor-inChief of this journal.

Open Access Statement: This is an Open Access article distributed in accordance with the Creative Commons Attribution-NonCommercial-NoDerivs 4.0 International License (CC BY-NC-ND 4.0), which permits the noncommercial replication and distribution of the article with the strict proviso that no changes or edits are made and the original work is properly cited (including links to both the formal publication through the relevant DOI and the license). See: https://creativecommons.org/licenses/by-nc-nd/4.0/.

\section{References}

1. Baumgartner H, Falk V, Bax JJ, De Bonis M, Hamm C, Holm PJ, Iung B, Lancellotti P, Lansac E, Muñoz DR, Rosenhek R, Sjögren J, Mas PT, Vahanian A, Walther T, Wendler O, Windecker S, Zamorano JL 2017 ESC/ EACTS Guidelines for the Management of Valvular Heart Disease. Eur Heart J 2017;38:2739-91.

2. Kleczyński P, Dziewierz A, Daniec M, Bagieński M, Rzeszutko Ł, Sorysz D, Trębacz J, Sobczyński R, Tomala M, Dudek D. Impact of post-dilatation on the reduction of paravalvular leak and mortality after transcatheter aortic valve implantation. Kardiol Pol 2017;75:742-8.

3. Kleczyński P, Zasada W, Bagieński M, Rzeszutko $€$, Sorysz D, Sobczyński R, Trębacz J, Kapelak B, Zembala M, Dudek D. Paravalvular leak after transcatheter aortic valve implantation (TAVI): Short-term results. Data from Polish national POL-TAVI registry. Cardiol J 2016;23:163-8.

4. Leon MB, Smith CR, Mack MJ, Makkar RR, Svensson LG, Kodali SK, Thourani VH, Tuzcu EM, Miller DC, Herrmann HC, Doshi D, Cohen DJ, Pichard AD, Kapadia S, Dewey T, Babaliaros V, Szeto WY, Williams MR, Kereiakes D, Zajarias A, Greason KL, Whisenant BK, Hodson RW, Moses JW, Trento A, Brown DL, Fearon WF, Pibarot P, Hahn RT, Jaber WA, Anderson WN, Alu MC, Webb JG. PARTNER 2 Investigators. Transcatheter or surgical aortic-valve replacement in intermediate-risk patients. N Engl J Med 2016;374:1609-20.

5. Qian Z, Wang K, Liu S, Zhou X, Rajagopal V, Meduri C, Kauten JR, Chang YH, Wu C, Zhang C, Wang B, Vannan MA. Quantitative Prediction of Paravalvular Leak in Transcatheter Aortic Valve Replacement Based on TissueMimicking 3D Printing. JACC Cardiovasc Imaging 2017;10:719-31.

6. Kodali SK, Williams MR, Smith CR, Svensson LG, Webb JG, Makkar RR, Fontana GP, Dewey TM, Thourani VH, Pichard AD, Fischbein M, Szeto WY, Lim S, Greason KL, Teirstein PS, Malaisrie SC, Douglas PS, Hahn RT, Whisenant B, Zajarias A, Wang D, Akin JJ, Anderson WN, Leon MB;the PARTNER Trial Investigators. Twoyear outcomes after transcatheter or surgical aortic valve replacement. N Engl J Med 2012;366:1686-95.

7. Généreux P, Head SJ, Hahn R, Daneault B, Kodali S, Williams MR, van Mieghem NM, Alu MC, Serruys PW, Kappetein AP, Leon MB. Paravalvular leak after transcatheter aortic valve replacement: the new Achilles' heel? A comprehensive review of the literature. J Am Coll Cardiol 2013;61:1125-36.

8. deSouza NM, Achten E, Alberich-Bayarri A, Bamberg F, Boellaard R, Clément O, Fournier L, Gallagher F, Golay X, Heussel CP, Jackson EF, Manniesing R, Mayerhofer ME, Neri E, O'Connor J, Oguz KK, Persson A, Smits M, van Beek EJR, Zech CJ;European Society of Radiology. Validated imaging biomarkers as decision-making tools in clinical trials and routine practice: current status and recommendations from the EIBALL* subcommittee of the European Society of Radiology (ESR). Insights Imaging 2019;10:87.

9. Doube M, Kłosowski MM, Arganda-Carreras I, Cordeliéres F, Dougherty RP, Jackson J, Schmid B, 
Hutchinson JR, Shefelbine SJ. BoneJ: free and extensible bone image analysis in ImageJ. Bone 2010;47:1076-9.

10. Kappetein AP, Head SJ, Généreux P, Piazza N, van Mieghem NM, Blackstone EH, Brott TG, Cohen DJ, Cutlip DE, van Es GA, Hahn RT, Kirtane AJ, Krucoff MW, Kodali S, Mack MJ, Mehran R, Rodés-Cabau J, Vranckx P, Webb JG, Windecker S, Serruys PW, Leon MB; Valve Academic Research Consortium (VARC)2. Updated standardized endpoint definitions for transcatheter aortic valve implantation: the Valve Academic Research Consortium-2 consensus document (VARC-2). Eur J Cardiothorac Surg 2012;42:S45-60.

11. Zoghbi WA, Asch FM, Bruce C, Gillam LD, Grayburn PA, Hahn RT, Inglessis I, Islam AM, Lerakis S, Little SH, Siegel RJ, Skubas N, Slesnick TC, Stewart WJ, Thavendiranathan P, Weissman NJ, Yasukochi S, Zimmerman KG. Guidelines for the Evaluation of Valvular Regurgitation After Percutaneous Valve Repair or Replacement: A Report from the American Society of Echocardiography Developed in Collaboration with the Society for Cardiovascular Angiography and Interventions, Japanese Society of Echocardiography, and Society for Cardiovascular Magnetic Resonance (published correction appears in J Am Soc Echocardiogr 2019 Jul;32(7):914917). J Am Soc Echocardiogr 2019;32:431-75.

12. Wiktorowicz A, Wit A, Dziewierz A, Rzeszutko L, Dudek D, Kleczynski P. A novel approach to quantification of aortic valve calcifications in patients undergoing transcatheter aortic valve implantation. Minerva Cardioangiol 2019;67:3-10.

13. Wiktorowicz A, Wit A, Dziewierz A, Rzeszutko L, Dudek $\mathrm{D}$, Kleczynski P. Calcium pattern assessment in patients with severe aortic stenosis via the Chou's 5-steps rule. Curr Pharm Des 2019;25:3769-75.

14. Merkus HG. Particle Size, Size Distributions and Shape. Particle Size Measurements. Particle Technology Series, vol 17. Springer, Dordrecht 2009.

15. Willson AB, Webb JG, Labounty TM, Achenbach S, Moss R, Wheeler M, Thompson C, Min JK, Gurvitch R, Norgaard BL, Hague CJ, Toggweiler S, Binder R, Freeman M, Poulter R, Poulsen S, Wood DA, Leipsic J. 3-Dimensional aortic annular assessment by multidetector computed tomography predicts moderate or severe paravalvular regurgitation after transcatheter aortic valve replacement: a multicenter retrospective analysis. J Am Coll Cardiol 2012;59:1287-94.

16. Condado JF, Corrigan FE 3rd, Lerakis S, Parastatidis I, Stillman AE, Binongo JN, Stewart J, Mavromatis K,
Devireddy C, Leshnower B, Guyton R, Forcillo J,Patel A, Thourani VH, Block PC, Babaliaros V. Anatomical risk models for paravalvular leak and landing zone complications for balloon expandable transcatheter aortic valve replacement. Catheter Cardiovasc Interv 2017;90:690-700.

17. Hamdan A, Guetta V, Klempfner R, Konen E, Raanani E, Glikson M, Goitein O, Segev A, Barbash I, Fefer P, Spiegelstein D, Goldenberg I, Schwammenthal E. Inverse relationship between membranous septal length and the risk of atrioventricular block in patients undergoing transcatheter aortic valve implantation. JACC Cardiovasc Interv 2015;8:1218-28.

18. Condado JF, Lerakis S, Stewart J, Jensen H, Henry TS, Ko SM, Stillman A, Rajaei MH, Mavromatis K, Devireddy C, Sarin E, Leshnower B, Guyton R, Kaebnick B, Thourani VH, Block PC, Babaliaros V. Balloon versus computed tomography sizing of the aortic annulus for transcatheter aortic valve replacement and the impact of left ventricular outflow tract calcification and morphology on sizing. J Invasive Cardiol 2016;28:295-304.

19. Wong DT, Bertaso AG, Liew GY, Thomson VS, Cunnington MS, Richardson JD, Gooley R, Lockwood S, Meredith IT, Worthley MI, Worthley SG. Relationship of aortic annular eccentricity and paravalvular regurgitation post transcatheter aortic valve implantation with CoreValve. J Invasive Cardiol 2013;25:190-5.

20. Corrigan FE 3rd, Gleason PT, Condado JF, Lisko JC, Chen JH, Kamioka N, Keegan P, Howell S, Clements SD Jr, Babaliaros VC, Lerakis S. Imaging for Predicting, Detecting, and Managing Complications After Transcatheter Aortic Valve Replacement. JACC Cardiovasc Imaging 2019;12:904-20.

21. Haensig M, Lehmkuhl L, Rastan AJ, Kempfert J, Mukherjee C, Gutberlet M, Holzhey DM, Mohr FW. Aortic valve calcium scoring is a predictor of significant paravalvular aortic insufficiency in transapical-aortic valve implantation. Eur J Cardiothorac Surg 2012;41:1234-40; discussion 1240-1.

22. Schultz CJ, Tzikas A, Moelker A, Rossi A, Nuis RJ, Geleijnse MM, van Mieghem N, Krestin GP, de Feyter P, Serruys PW, de Jaegere PP. Correlates on MSCT of paravalvular aortic regurgitation after transcatheter aortic valve implantation using the Medtronic CoreValve prosthesis. Catheter Cardiovasc Interv 2011;78:446-55.

23. Vahanian A, Alfieri O, Al-Attar N, Antunes M, Bax J, Cormier B, Cribier A, De Jaegere P, Fournial G, Kappetein AP, Kovac J, Ludgate S, Maisano F, Moat N, 
Mohr F, Nataf P, Piérard L, Pomar JL, Schofer J, Tornos P, Tuzcu M, van Hout B, Von Segesser LK, Walther T; European Association of Cardio-Thoracic Surgery; European Society of Cardiology; European Association of Percutaneous Cardiovascular Interventions. Transcatheter valve implantation for patients with aortic stenosis: a position statement from the European Association of Cardio-Thoracic Surgery (EACTS) and the European Society of Cardiology (ESC), in collaboration with the European Association of Percutaneous Cardiovascular Interventions (EAPCI). Eur Heart J 2008;29:1463-70.

24. Larroche J, Panh L, Lhermusier T, Bataille V, Marachet MA, Chollet T, Petermann A, Bouisset F, Boudou N, Marcheix B, Rousseau H, Galinier M, Carrié D, Lairez O, Lavie-Badie Y. Impact of aortic valve calcification severity on device success after transcatheter aortic valve replacement. Int J Cardiovasc Imaging 2020;36:731-40.

25. Alqahtani AM, Boczar KE, Kansal V, Chan K, Dwivedi G, Chow BJ. Quantifying Aortic Valve Calcification using Coronary Computed Tomography Angiography. J Cardiovasc Comput Tomogr 2017;11:99-104.

26. Ryś M, Hryniewiecki T, Michałowska I, Stokłosa P, Różewicz-Juraszek M, Chmielak Z, Dąbrowski M, Mirota K, Szymański P. Quantitative estimation of aortic valve calcification in multislice computed tomography in predicting the development of paravalvular leaks following transcatheter aortic valve replacement. Postepy Kardiol Interwencyjnej 2018;14:85-9.

27. Koh EY, Lam KY, Bindraban NR, Cocchieri R, Planken RN, Koch KT, Baan J Jr, de Mol BA, Marquering HA. Aortic valve calcification as a predictor of location and severity of paravalvular regurgitation after transcatheter aortic valve implantation. Interact Cardiovasc Thorac Surg 2015;20:345-50.

Cite this article as: Wiktorowicz A, Wit A, Malinowski KP, Dziewierz A, Rzeszutko L, Dudek D, Kleczynski P. Paravalvular leak prediction after transcatheter aortic valve replacement with self-expandable prosthesis based on quantitative aortic calcification analysis. Quant Imaging Med Surg 2021;11(2):652664. doi: 10.21037/qims-20-669
28. Bianchi M, Marom G, Ghosh RP, Rotman OM, Parikh P, Gruberg L, Bluestein D. Patient-specific simulation of transcatheter aortic valve replacement: impact of deployment options on paravalvular leakage. Biomech Model Mechanobiol 2019;18:435-51.

29. Obaid DR, Smith D, Gilbert M, Ashraf S, Chase A. Computer simulated "Virtual TAVR" to guide TAVR in the presence of a previous Starr-Edwards mitral prosthesis. J Cardiovasc Comput Tomogr 2019;13:38-40.

30. Luraghi G, Migliavacca F, García-González A, Chiastra C, Rossi A, Cao D, Stefanini G, Rodriguez Matas JF. On the Modeling of Patient-Specific Transcatheter Aortic Valve Replacement: A Fluid-Structure Interaction Approach. Cardiovasc Eng Technol 2019;10:437-55.

31. Dowling C, Bavo AM, El Faquir N, Mortier P, de Jaegere P, De Backer O, Sondergaard L, Ruile P, Mylotte D, McConkey H, Rajani R, Laborde JC, Brecker SJ. PatientSpecific Computer Simulation of Transcatheter Aortic Valve Replacement in Bicuspid Aortic Valve Morphology. Circ Cardiovasc Imaging 2019;12:e009178.

32. Pawade T, Sheth T, Guzzetti E, Dweck MR, Clavel MA. Why and How to Measure Aortic Valve Calcification in Patients With Aortic Stenosis. JACC Cardiovasc Imaging 2019;12:1835-48.

33. Khalique OK, Hahn RT, Gada H, Nazif TM, Vahl TP, George I, Kalesan B, Forster M, Williams MB, Leon MB, Einstein AJ, Pulerwitz TC, Pearson GD, Kodali SK. Quantity and location of aortic valve complex calcification predicts severity and location of paravalvular regurgitation and frequency of post-dilation after balloon-expandable transcatheter aortic valve replacement. JACC Cardiovasc Interv 2014;7:885-94. 